\title{
PARALELOS EN LA MUSIVARIA ROMANA DE GRECIA E HISPANIA. A PROPÓSITO DE UN MOSAICO DE ALCOLEA DEL RÍO Y UN PAVIMENTO DE MITILENE.
}

\author{
LUz NEIRA JIMÉNEZ \\ CSIC-Universidad Carlos III de Madrid
}

\section{Resumen}

El presente artículo aborda las circunstancias del descubrimiento y los avatares posteriores de un mosaico hallado en Alcolea del Río (Sevilla), cuyos fragmentos se conservan en la Hispanic Society of America. El estudio de su composición refleja evidentes relaciones con otros mosaicos romanos, aunque el paralelo más estrecho se encuentra en un fragmentario pavimento de Mitilene (Lesbos), para el que se propone una hipótesis de reconstrucción.

\section{Summary}

This article is a study of the discovery and history of a roman mosaic found in Alcolea del Río (Sevilla), whose fragments are preserved in the Hispanic Society of America. The study of the composition shows us relations with other roman mosaics. However, the most direct paralell is a pavement found in Mytilene (Lesbos), which I proposed a hypothesis of reconstruction.

Con motivo del VI Coloquio Internacional sobre Mosaico Antiguo celebrado en Palencia y Mérida presentamos una comunicación sobre el mosaico de los tritones hallado en Santiponce, que se conserva en el Museo Arqueológico de Sevilla (NEIRA, 1994, 359-367), donde, entre otras cuestiones, se ponía de manifiesto que su esquema compositivo, es decir, la disposición sobre los ángulos de un mosaico de cuatro tritones, orientados de cara al exterior, formando el thiasos marino de una representación central, estaba también documentado en otros ejemplares de la musivaria romana. Entre los paralelos considerados figuraban citados cuatro mosaicos bícromos - dos hallados con seguridad en Ostia, un tercero que fue trasladado a fines del siglo XVIII a Zarskoje Sselo, aunque de probable procedencia también ostiense, o al menos de la órbita campana, y el cuarto de Roma - así como un mosaico polícromo del Norte de África. No 
obstante, apuntábamos que el mosaico italicense, encontrado en la calle Pescadores de Santiponce, no debió figurar como único exponente de este esquema en la musivaria hispana, a juzgar por la mención de una representación similar, que aparece recogida en una obra publicada a fines del siglo XIX (CAMPOS, 1897,16), donde su autor manifestaba textualmente «en el Museo de Carmona me han dicho sus ilustrados directores, que se conserva un pequeño mosaico descubierto en Alcolea del Río (que según el Sr. Bonsor fue la antigua Canama), con tritones en igual disposición que los del Museo provincial, conteniendo en el centro una cabeza de Medusa».

Nuestros primeros intentos en la búsqueda del citado mosaico de Alcolea del Río (Sevilla) resultaron infructuosos, ya que el pavimento no se halla ni en el Museo de Carmona, fundado bajo los auspicios de la Sociedad Arqueológica de Carmona (MAIER, 1997, 303-310) y gracias a la incansable actividad, entre otras figuras de la época, de Bonsor, ni en el Castillo de Mairena, donde a partir de principios de siglo fue trasladada una buena parte de la colección perteneciente al arqueólogo de origen anglo-francés. Sin embargo, siguiendo la pista de Bonsor y su relación con diversos eruditos y apasionados por las antigüedades que viajaban a la España de finales del siglo XIX con el propósito de visitar los lugares de mayor expectación arqueológica como Italica y Carmona, conocemos la amistad entablada con Archer M. Huntington, heredero norteamericano de una gran fortuna que, ya de regreso a su país, fundaría a principios de este siglo la Hispanic Society of America y a quien Bonsor, con el fin de financiar las obras de remodelación y acondicionamiento de su nueva casa-museo, el citado Castillo de Mairena, vendió un gran número de piezas, entre las que se cuenta el mosaico de Alcolea del Río, mencionado y reproducido en un catálogo de la Hispanic en 1938 (The Hispanic Society of America. Handbook, 1938, 106).

No obstante, una vez constatada la conservación y localización del mosaico, que pasó a formar parte de la colección de la Hispanic Society of America en octubre de $1905^{1}$, la cuestión fundamental era investigar el lugar y la fecha exacta de su descubrimiento, así como las circunstancias que propiciaron su traslado desde el museo de Carmona hasta la Hispanic Society en Nueva York, donde figura descubierto por Bonsor en la mencionada población de Alcolea del Río. Partiendo de la fecha de edición de la obra de Campos, que sitúa al mosaico expuesto en el Museo de Carmona ya en 1897, las publicaciones de Bonsor sobre su actividad arqueológica en los años anteriores a 1897 no son demasiado esclarecedoras, quizá porque la excavación de la necrópolis de Carmona (BENDALA, 1976) centraba el máximo interés en detrimento de otros hallazgos. De hecho, la primera publicación de Bonsor en hacerse eco del mosaico (BONSOR, 1901,

\footnotetext{
${ }^{1}$ Según nos informó en su carta del 25 de enero de 1994 la Dra. Vivían A. Hibbs, Curator of Archaeology de la Hispanic Society of America, a quien queremos hacer constar nuestro agradecimiento.
} 
855) es posterior en algunos años a la cita de Campos y tan sólo se menciona en una escueta relación de antigüedades, procedentes de Alcolea, que habían sido enviadas al Museo de la Necrópolis de Carmona, tras una más detallada información sobre una inscripción hallada en 1888, que fijaba el nombre antiguo de Alcolea del Río como Canania, y sobre los restos de un pequeño edificio romano, un depósito de agua de forma circular y cubierto por una bóveda semiesférica, conocido como la Bola. Concretamente, Bonsor mencionaba en la lista de antigüedades dos urnas de vidrio en su funda de plomo, enterradas dentro de una media ánfora, el mosaico con la cabeza de Medusa en el centro y tritones en los ángulos, un fragmento de estatua, una tablilla de plomo en la que se ve en relieve una mujer que lleva una guirnalda -indicando que se trataba de una donación del Sr. Engel al Museo- y un capitel de mármol blanco. Ninguna referencia explícita al contexto en el que fueron descubiertas las piezas ni por supuesto al año o a las circunstancias de su hallazgo.

En una obra ya más extensa, mucho más posterior, sobre la Expedición Arqueológica del Guadalquivir (1889-1901) (BONSOR, 1931, 1-2, 41-42), se encuentran párrafos más clarificadores al detallar que los trabajos en las riberas del Guadalquivir, con la intención de identificar los oppida mencionados por Plinio y entre los que se nombraba Canama, se habían comenzado en compañía de Mr. Clark-Maxwell, de Cambridge, a mediados de diciembre de 1889 y habían sido concluidos a finales de abril de 1890. Del mismo modo que en su artículo publicado en la Revista de Archivos, Bibliotecas y Museos de 1901, Bonsor agregaba después al referirse a Alcolea del Río y a las piezas que habían sido trasladadas al Museo de la Necrópolis la misma relación de antigüedades, por lo que es lógico suponer que éstas procederían de los trabajos de excavación llevados a cabo en el periodo citado, entre finales de 1889 y abril de 1890.

Sin embargo, la información más reveladora se halla en las Actas de la Sociedad Arqueológica de Carmona, cuyos manuscritos se conservan en el Museo Arqueológico de Sevilla ${ }^{2}$. Concretamente en las Actas de la sesión ordinaria del 26 de abril de 1890 se recoge que el Sr. Bonsor manifestó a la Sociedad «haber terminado la exploración arqueológica de los despoblados comprendidos en los lados del Guadalquivir entre Córdoba y Sevilla y cuya exploración, levantamiento de planos y descripción de los monumentos encontrados con tal motivo serán objeto de una memoria que en unión de Mr. Clark publicará dentro de breve tiempo, manifestó así mismo algunas particularidades dignas de mencionarse como son rectificación de los nombres de algunos pueblos de la ribera del Guadalquivir citados por Plinio asignando el sitio que a su juicio éstos debían ocupar. Que durante las excavaciones practicadas en la

\footnotetext{
${ }^{2}$ Deseamos agradecer a su director, D. Fernando Fernández, todas las facilidades dadas para consultar los citados manuscritos.
} 
Peña de la Sal (Municipio Flavio Arvense) se habían encontrado pinturas murales, mosaicos con dibujos, ánforas, plomos con figuras de relieves, un Hermes de mármol sin cabeza y una cabeza de Baco admirablemente trabajada. De estos objetos las pinturas murales que representan un cuadro de asunto erótico, el mosaico y una plancha de plomo que representa una mujer vestida que marcha llevando en sus manos una guirnalda corta han sido regalados al Museo de la Necrópolis por los señores Mr. Arthur Engel y Mr. Clark compañeros de la sociedad que a este objeto habían constituido con el Sr. Bonsor para hacer las excavaciones en el sitio mencionado, que tanto el Sr. Clark como el Sr. Engel habían encargado al Sr. Bonsor presentara a la sociedad sus respetos y afectos de despedida».

Aunque en el manuscrito no se especifica el tipo de figuras representadas en el mosaico, -la Medusa y los tritones-, la mención de la plancha de plomo con representación de una mujer portando una guirnalda, que también aparecerá citada en la relación de antigüedades incluida en sus obras posteriores (BONSOR, 1901; IDEM, 1931), no parece dejar dudas sobre la identificación del mosaico con el ejemplar objeto de nuestro estudio. Podría, no obstante, resultar chocante la omisión del nombre de Alcolea del Río como lugar de procedencia de los hallazgos, máxime si tenemos en cuenta que dicho nombre será el que figure sistemáticamente en las publicaciones posteriores. Sin embargo, es digno de precisar que La Peña de la Sal, el lugar citado en las Actas de la Sociedad correspondientes a la sesión ordinaria del 26 de abril de 1890, pertenecía al término municipal de Alcolea del Río, según se hace constar, entre otros, en la portada de las Actas relativas a la tercera sesión extraordinaria del 23 de octubre de 1885 de la Sociedad Arqueológica de Carmona, donde se daba cuenta de la segunda Excursión de la Sociedad, que había tenido como destino la Peña de la Sal (Alcolea).

Podemos situar, por tanto, la fecha del hallazgo entre finales de 1889 y principios de 1890 y su procedencia de la Peña de la Sal en el término municipal de Alcolea del Río, aunque desgraciadamente seguimos desconociendo el lugar que pavimentaba y, en consecuencia, su contexto arqueológico. Sí sabemos, en cambio, que el mosaico debió ser trasladado muy pronto al Museo de la Necrópolis de Carmona, que a pesar de ser inicialmente fundado en 1885 para albergar los materiales procedentes de las excavaciones de la propia necrópolis fue también sede de una gran colección de antigüedades halladas en el transcurso de varias excursiones organizadas por la Sociedad y de algunas excavaciones dirigidas por Bonsor en otros yacimientos.

A juzgar por la noticias transmitidas por el periódico La Andalucía, en su edición de 25 de mayo de 1885, con motivo precisamente de la inauguración de la primera sede del museo en un piso alto de un colegio de Carmona, varios mosaicos colocados en marcos de madera se exponían ya como parte de la colección inicial y, en este 
sentido, la incorporación del llamado mosaico de Alcolea ya a la nueva y definitiva sede del museo en terrenos de la Necrópolis, concluida en junio de 1888, no debió resultar demasiado chocante entre más de una veintena de fragmentos de mosaico, que figuraban citados en aquel primer museo de carácter temporal.

Por tanto, en el Museo de la Necrópolis de Carmona el mosaico estuvo expuesto desde 1890 hasta 1905, fecha en la que fue vendido y enviado a la Hispanic Society. Sobre las conversaciones previas, claro está por correspondencia, que culminaron en la venta del mosaico, son muy reveladoras las cartas intercambiadas entre el propio Bonsor, por una parte, y Archer M. Huntington y algunas personas de su entorno, por otra, que han sido traducidas e incluidas en la reciente tesis doctoral de J. Maier ${ }^{3}$ sobre la personalidad de Bonsor (MAIER, 1997a, núms. 15-31). Son fruto de una amistad iniciada tras coincidir en Itálica en 1898, adonde Huntington había acudido atraído por los restos descubiertos tras llegar a España seis años antes y en cuyo lugar Bonsor se encontraba con el citado M. Engel. La primera carta en la que se habla del mosaico está fechada el 10 de febrero de 1905, mientras que la última data del 15 de septiembre de ese mismo año. Quizás una de las más interesantes es aquella misiva del 18 de febrero, en la que Bonsor transmite a Huntington que acaba de llegar a un arreglo (sobre los mosaicos) con su socio (Juan Fernández López, Secretario de la Sociedad Arqueológica de Carmona), después de largas y arduas discusiones y le promete enviarle fotografías de las partes más importantes de un interesante mosaico que está en el Museo y que les gustaría vender. En carta del 9 de abril Bonsor le confirma el envío de dibujos de las piezas con diseños geométricos y fotografías de las que contienen figuras y menciona que los dos mosaicos objeto de interés fueron descubiertos hace 10 o 12 años en Alcolea del Río, valorando el denominado como $\mathrm{n}^{\circ} 1$, el de la medusa y tritones en 5 fragmentos, en 4.000 pesetas y el $\mathrm{n}^{\circ} 2$ (Diana y 26 piezas) en 1.500 pesetas. Añade, además, que a pesar de haber intentado una rebaja de su socio (Juan Fernández López) para el $n^{\circ} 1$ no ha podido conseguirlo, ya que «las cuatro partes completas han estado muchos años colocadas en las paredes del Museo» y «Están tan hermosos con sus marcos negros que él (Fernández) no parece estar muy dispuesto a desprenderse de ellos».

Esta carta revela datos importantes sobre el mosaico, aunque presenta algunos datos contradictorios. Por un lado, nos transmite el precio de venta, 4.000 pesetas de aquella época, sin duda, una suma considerable, así como la costumbre de exponer los mosaicos a modo de cuadros en marcos negros igual que aquella veintena de fragmentos citados en el primer museo de Carmona. Sin embargo, resulta extraño que el propio Bonsor

\footnotetext{
${ }^{3}$ Quede constancia en estas líneas de nuestro agradecimiento al Dr. Jorge Maier Allende por habernos permitido consultar su tesis doctoral.
} 
indique que los dos mosaicos habían sido descubiertos en Alcolea hace 10 o 12 años, lo que correspondería a 1895 o 1893, años en los que no se tiene noticia de ninguna actividad arqueológica de Bonsor en Alcolea, aunque después añada que el apego de su socio por el mosaico de la medusa está fundamentado en los muchos años que sus cuatro partes completas habían estado colocadas en las paredes del Museo.

Tras recibir esta carta y los dibujos de los mosaicos, Huntington no se hace esperar y, aún expresando lo elevado de la suma, manifiesta en una misiva fechada el 14 de abril su aceptación al precio solicitado por Bonsor. El envío, no obstante, se retrasa por el padecimiento de una gripe que tiene a Bonsor más de un mes alejado de toda actividad, tal y como refiere el arqueólogo residente en Carmona en su correspondencia del 4 de junio. En estas fechas, ya repuesto de la enfermedad, comunicará a Huntington que por fin ha podido ocuparse del embalaje de las 13 cajas, que ya están en camino, y dos semanas más tarde se pone en contacto con el Sr. Hillhouse (del personal adscrito a la Hispanic Society of America) para asegurarle que el día 17 (de junio) había enviado vía Liverpool las 13 cajas con mosaicos romanos, incluyendo facturas de conocimiento de embarque y del consulado. Ausente de Nueva York por encontrarse en Inglaterra, Hillhouse escribe a Bonsor el 31 de julio de 1905, respondiendo que las 13 cajas de mosaicos han llegado a salvo a Nueva York, aunque todavía no las han recibido de la casa de aduanas. Esta misiva debió cruzarse con otra de Bonsor fechada el 2 de agosto en París y destinada a Huntington, donde se expresaba la esperanza de que las 13 cajas habrían llegado ya a su destino, al tiempo que se notifica que la semana siguiente saldrían desde Sevilla otras dos cajas más con una pequeña colección de antigüedades de la Necrópolis Romana de Carmona y una pieza del mosaico que se había quedado atrás por equivocación. Por fin, el 24 de agosto Huntington contestará a Bonsor, comentándole que tiene entendido que las cajas con mosaicos han llegado, aunque todavía no las había examinado al no hallarse en Nueva York. El envío de un cheque en libras por el equivalente a 12.200 pesetas en pago por dos cajas de antigüedades de Carmona (6.700) y las 13 cajas con mosaicos romanos (5.500) fue comunicado a Bonsor por el tesorero de la Hispanic Society en una carta del 15 de septiembre, quedando finalmente cerrada la operación de venta y traslado del mosaico de Alcolea del Río.

Un montaje con el dibujo del esquema geométrico del mosaico y las fotografías de los cinco fragmentos mencionados que Bonsor envió a Huntington con el fin de que el fundador de la Hispanic Society pudiera contemplar las piezas que iba a adquirir figura expuesto en el Museo neoyorquino, según la hipótesis de reconstrucción del propio Bonsor (Fig. 1). A tenor de dicho montaje, nos planteamos si los fragmentos trasladados a Nueva York fueron los únicos que se habían extraído en el momento de su descubrimiento o si, por el contrario, son el resultado de una selección, bien mediatizada por el mayor valor, en aquella época, de los motivos figurados, bien por las circunstancias del 
traslado. A este respecto, el informe que recogen las Actas de la Sociedad Arqueológica de Carmona en sesión ordinaria del 26 de abril de 1890 menciona el regalo y traslado al Museo del mosaico, sin especificar que se trate de sus fragmentos figurados. En el mismo sentido, Bonsor en su carta del 18 de febrero a Huntington manifiesta su intención de «enviarle las fotografías de las partes más importantes de un interesante mosaico romano que tenemos en el Museo y que nos gustaría vender», frase ambigua de la que podría sobreentenderse que el mosaico se conservaba íntegro, si bien sólo enviaría fotos de las partes más sobresalientes, o, por el contrario, que únicamente poseían los fragmentos más importantes del pavimento, aquellos que contenían motivos figurados. Sin embargo, en una misiva posterior, fechada el 9 de abril, Bonsor habla de piezas, piezas que pertenecieron a dos mosaicos descubiertos en Alcolea del Río... e incluso dice textualmente sobre el mosaico $\mathrm{n}^{\circ} 1$ (el de la Medusa) «Como verá Ud. por la restauración que propongo (dibujado a tinta) medía 2 metros cuadrados...», cita cinco piezas al valorar su venta y confirma su carácter fragmentario al referirse al apego de su socio por el mosaico y mencionar que llevaban mucho tiempo expuestos en el museo «tan hermosos con sus marcos negros».

Estas líneas apuntan también la posibilidad de que Bonsor no hubiera estado presente en la excavación del mosaico al revelar él mismo una hipótesis sobre su superficie y no datos exactos sobre las dimensiones del pavimento, quizás descubierto por sus colegas Clark o Engel, de los que sabemos por las Actas que participaron con él en la expedición llevada a cabo entre finales de 1889 y principios de 1890. Este hecho explicaría además la ausencia de noticias sobre el contexto arqueológico del mosaico que se advierte en los informes y escritos de Bonsor.

Esta circunstancia no resta valor a la hipótesis de reconstrucción de Bonsor, cuyas fotografías son de gran importancia para una posible reconstitución ideal del mosaico. En este sentido, las fotos de la época revelan el estado de los cinco fragmentos que estuvieron durante más de una década expuestos en el Museo de la Necrópolis de Carmona y nos sirven de referencia en el análisis de los fragmentos conservados. De este modo, la reconstrucción de Bonsor evidencia dos variaciones. Por un lado, daba fe de la existencia, aunque fragmentaria, de un tritón dotado de aletas natatorias que, en el ángulo superior derecho según la orientación de la Medusa, figuraba afrontado a otro tritón de idénticas características, y, por otro lado, pone de manifiesto la transformación del fragmento situado en el centro, que en la actualidad muestra en torno a la cabeza de Medusa inscrita en un círculo sólo el primero de los anillos concéntricos que decoraban el gran círculo predominante en la composición, formado ahora por 24 triángulos (lám. 1), mientras que la fotografía antigua muestra triángulos de hasta cuatro anillos, de un total estimado en 7, y revela un número de 28 triángulos -no 24en el primer anillo. 
Se trata, por tanto, de una composición geométrica que, bien documentada en la musivaria romana, se denomina roseta de triángulos curvilíneos. Este motivo se caracteriza por la disposición de anillos concéntricos compuestos por el mismo número de triángulos y cuya regla esencial es el aumento progresivo de su tamaño desde el interior hasta el exterior de la roseta. Aparece antes del cambio de Era en Pompeya (OVADIAH, 1980, 144) y es frecuente en la musivaria itálica alto-imperial, especialmente ostiense (BECATTI, 1961, núms. 153 y 423, láms. LXX-LXXI), aunque su difusión y su pervivencia se aprecia en pavimentos tardíos como el hallado en Héracléa Lynkestis, fechado en la segunda mitad del siglo VI d.C. (TOMASEVIC, 1975, lám. CLXXXVII, 2). En Hispania se documenta, además de en Alcolea del Río, en mosaicos, particularmente de los siglos II y III, de Ampurias, Itálica (LUZON, 1988, núms. 17 y 19), Badalona (BARRAL I ALTET, 1978, 83), Linares (BLAZQUEZ, 1981, núm. 50, lám. 58) y en varios de Mérida (BLANCO 1978, núms. 1, 32, 46 y 54), así como en un mosaico recientemente descubierto en Ecija $(A A A, 1998)$ y en un pavimento de Carmona (BLAZQUEZ, 1982, núm. 15, lám. 11), en cuyo centro también destaca la cabeza de Medusa. Asimismo, la roseta de triángulos curvilíneos aparece frecuentemente representada, entre otros, en los mosaicos romanos de Grecia, donde en muchos casos responde a una rica policromía, interpretada como característica propia del denominado grupo oriental (LUZON, 1988, 231, núms. 1-4 y 7). Sin embargo, quizás una de las notas más llamativas de los ejemplares hallados en Grecia sea la relativa frecuencia con la que una cabeza de Medusa figura decorando el círculo central, tanto en los mosaicos polícromos procedentes del Pireo y Corinto (LUZON, 1988, núms. 1-2, figs. 5-6), como en otros cuatro de rosetas bícromas, dos descubiertos en Patras, uno en Cnossos y otro en Thasos (PANAGIOTOPOULOU, 1994, 370371, figs. 4, 6-7).

Evidentemente, no en todos los mosaicos se reproduce el mismo número de anillos ni de triángulos, aunque un estudio detenido de este tipo de composición (LUZON, 1988, 213-241) revela la frecuente repetición de determinados números, así como la proporción entre el número de triángulos -16 y 32, o 12, 24 y 48- y de anillos concéntricos, poniendo de manifiesto que el dibujo de la roseta estaba sometido a unas reglas, probablemente con unas medidas fijas, y no en función de las dimensiones del pavimento a decorar.

Sin embargo, es también significativo el porcentaje de mosaicos en los que la roseta está formada por otro número de triángulos como en Alcolea, donde figuran 28 igual que en un mosaico polícromo de Roma (Settecamini) (LUZON, 1988, 231) y en otro bícromo de la casa de Apuleyo en Ostia (BECATTI, 1961, núm. 153, lám. LXX), éste último también con la cabeza de Medusa en el centro, aunque tanto en uno como en otro varía el número de anillos concéntricos, 14 en Roma y 11 en el ostiense. 
En cuanto a los motivos incluidos en los ángulos resultantes de la inscripción del gran círculo decorado con la roseta en el campo cuadrado de los mosaicos citados se aprecia una gran diversidad, desde motivos geométricos y vegetales hasta figurados como los bustos de las cuatro Estaciones. En Alcolea del Río, en cambio, fueron elegidas las figuras de cuatro tritones, afrontados e idénticos dos a dos y dispuestos de cara al exterior. Se trata de tritones jóvenes e imberbes, según la tónica característica de los representados en la musivaria hispana (NEIRA, 1991, 513-529) que, aún avanzando de perfil bien hacia la izquierda o hacia la derecha, presentan su torso humano de tres cuartos y están dotados de una sinuosa cola pisciforme con aleta caudal que se adapta a la superficie del mosaico.

Según la orientación de la Medusa, los tritones situados en los ángulos inferiores van provistos de extremidades anteriores equinas y pertenecen, por tanto, a la variedad de tritones más representadas en la musivaria romana, la del centauro marino (NEIRA, 1992, 223 y ss.). El de la izquierda (lám. 2), que al aparecer afrontado a su paredro figura lógicamente avanzando hacia la derecha, porta en su mano izquierda, con el brazo flexionado, el extremo de un remo dispuesto en diagonal sobre su hombro derecho y extiende hacia delante la mano derecha, sosteniendo un pequeño delfín, al tiempo que torna ligeramente su cabeza hacia atrás, vista de tres cuartos ${ }^{4}$, del mismo modo que el centauro marino situado en el ángulo inferior derecho (lám. 3), representado hacia la izquierda y con los mismos atributos pero en mano inversa. Según nuestro estudio tipológico (NEIRA, 1992, 163-170), del que se desprenden 6 tipos, estas representaciones de tritones corresponden al tipo 4, aunque en esta posición la mayoría aparece tocando un instrumento musical, guiando las riendas de un monstruo marino o sosteniendo la concha de la Venus marina. Su distinta actitud, portando dos atributos, concretamente el alargado remo, más propios de otro tipo muy difundido, se enmarca sin duda en la gran diversidad que, aún dentro de una serie de reglas comunes, caracteriza a este género de representaciones. Llama la atención, no obstante, la disposición del remo sobre el hombro inverso, cuando suele ser más frecuente que éste $\mathrm{u}$ otros atributos de forma similar descansen sobre el mismo hombro.

Con independencia de su disposición poco ortodoxa, el remo es uno de los atributos de origen marino más representado tras la caracola y el timón de espadilla. No así el pequeño delfín que, siendo como el tridente un atributo relacionado con el inicial carácter divino de Tritón, entre otras divinidades marinas, figurará en la musivaria romana más comúnmente asociado a Poseidón/Neptuno y sólo perdurará en algunas representaciones de tritones (NEIRA, 1992, 270-271) en mosaicos bícromos de

${ }^{4}$ Este tritón aparece reproducido en una breve y reciente nota sobre las piezas conservadas en la Hispanic Society (THEODOROU, 1991, fig. 6). 
Herculano (MAIURI, 1958, 105, figs. 79-81), Isthmia (PACKARD, 1980, láms. 97101) y Palazzo Farnese de Roma (BROISE ETALII, 1977, figs. 34 y 37) o en polícromos de Conimbriga (NEIRA 1991, fig. 2) y Neapolis (DARMÓN, 1980, 43) 5 .

A juzgar por la fotografía del fragmento superior derecho del mosaico, que contenía la figura de un tritón al que le falta la cabeza y su hombro derecho, y en virtud del tritón conservado (lám. 4), en los ángulos superiores se situaban en cambio dos tritones dotados de aletas natatorias a modo de extremidades anteriores, -variedad que por su número es la segunda más documentada en los mosaicos romanos (NEIRA, 1992, 223). Ambos respondían al tipo denominado 1, el más numeroso entre las representaciones atestiguadas en la musivaria romana, que se caracteriza por figurar avanzando hacia la izquierda o la derecha, con el torso visto de tres cuartos, mientras con un brazo flexionado, el izquierdo si avanzan en esa dirección, presentan una mano a la altura de la cadera y extienden la otra, la derecha, hacia delante (NEIRA, 1992, 141152). Los dos tritones muestran además los atributos más frecuentemente portados, un timón de espadilla y una caracola. Se trata de la representación más convencional y heráldica, ya que ambos sujetan con la mano situada a la altura de cadera la vara del timón que descansa de modo ortodoxo sobre el mismo brazo, al tiempo que, con la cabeza de tres cuartos y el brazo extendido en el sentido de la marcha, aparecen soplando una caracola, cuyo extremo sostienen en la otra mano.

En las cuatro representaciones, las colas pisciformes de los tritones ascienden sinuosamente por los laterales, ciñéndose perfectamente al espacio angular. En este sentido, al estudiar la disposición de cuatro tritones orientados de cara al exterior sobre los ángulos del campo de un mosaico (NEIRA, 1994, 364-366) ya señalábamos la propicia adecuación de estas figuras, así como la evolución de la composición. A este respecto, cabe apuntar que las figuras de cuatro tritones situados en los ángulos de un mosaico se documentan inicialmente, en compañía de otros miembros habituales de un thiasos marino como son las nereidas representadas sobre animales marinos en los lados y en torno a una representación central de Scylla, en un pavimento de las termas de Neptuno en Ostia, del 139 d.C. (BECATTI, 1961, núm. 71). Igualmente cuatro tritones figuraban en las esquinas de otro mosaico bícromo hallado en Sentinum (MORETTI, 1925, figs. 1-2), aunque en este pavimento son diversos monstruos marinos los que decoraban tanto los lados como el centro del campo sin

\footnotetext{
${ }^{5}$ Véase más adelante en lo relativo a un mosaico de Mitilene. Sin relación con este tipo de representaciones un delfín ya de considerables dimensiones figura apresado por un centauro marino en el pavimento fragmentario de El Chorreadero, quien lo mantiene con su mano izquierda contra su cuerpo (BLAZQUEZ, 1982, núms. 48-49, láms. 20-21); mientras tanto en los mosaicos también bícromos de Porta Collina (NEIRA 1996, 227-228, fig. 4) y.Tor di Quinto (AURIGEMMA, 1958, núm. 340) un delfín de similares proporciones al bético está representado en el instante de lanzarse sobre la enroscada cola pisciforme de un tritón.
} 
aparente orden, y un esquema relativamente similar al ostiense se advierte en un fragmentario pavimento también bícromo de Mérida, de la primera mitad del siglo 111 d.C. (BLANCO 1978, núm. 7), donde, no obstante, los tritones situados en las esquinas al no figurar afrontados ocupan el lado inferior del mosaico y probablemente el superior, mientras nereidas sobre animales marinos decoraban los laterales, en torno a la representación central del triunfo de Neptuno (NEIRA, 1996b, tav. XIV,1).

En otros pavimentos de mediados del siglo II y principios del III puede apreciarse en cambio como los cuatro tritones acaban por erigirse en únicos miembros de un cortejo dispuesto en torno a una representación central, concretamente en otro mosaico bícromo y también itálico que fue trasladado a Zarskoje Sselo (KORSUNSKA, 1928, 360-371, fig. 1, lám. 12), en un pavimento de las Termas Marítimas de Ostia (BECATTI, 1961, núm. 212), en el citado mosaico polícromo hallado en Santiponce 6 (NEIRA, 1994, 359-367) y en otro ejemplar del Norte de África (FOUCHER, 1965, 142, nota 27, fig. 3). En estos mosaicos tanto los tritones como la representación central componen la decoración del campo del mosaico, que no presenta otros motivos ni compartimentación alguna.

El mosaico de Alcolea se distingue de los mosaicos citados al presentar los tritones no directamente en torno a la cabeza de Medusa, sino en los espacios resultantes de la inscripción del círculo, decorado con la roseta de triángulos curvilíneos, en el campo cuadrado. Sin embargo lejos de constituir un hapax, esta representación documenta una tendencia que, a juzgar por la existencia de algunos paralelos, también debió gozar de cierta difusión en la musivaria romana. En este sentido, las noticias del descubrimiento de un mosaico a fines del siglo XIX en Villa Casali, en los alrededores de Roma, nos refieren la representación de cuatro colosales tritones sobre los ángulos de un campo decorado con diversos peces en torno a un círculo central delimitado por una greca, dentro del cual un disco de cipollino habría sustituido ya desde antiguo a otro de un finísimo mosaico policromo, de principios del siglo III d.C. (LANCIANI, 1885, 341, 422; BLAKE, 1940, 95). Desconocemos las dimensiones del mosaico, hoy perdido, y de las figuras, lo que nos impide asegurar si dicho círculo poseía un reducido diámetro y enmarcaba únicamente la representación central, de modo más cercano a los ejemplares antes citados, o si, por el contrario, mostraba una entidad semejante a la del mosaico de Alcolea. Una relación evidente si se aprecia en un mosaico polícromo hallado en Trier, que ha sido fechado en el primer cuarto del siglo IV d.C. (PARLASCA, 1959, 55-56, lám. 53). A pesar del deterioro que presentaba ya en la época del descubrimiento y de su actual estado fragmentario, el mosaico

\footnotetext{
${ }^{6}$ Uno de los grabados más antiguos fue realizado por Demetrio de los Ríos y ha sido recientemente expuesto y reproducido en Las Excavaciones de Itálica. Los dibujos de Demetrio de los Ríos..Sevilla 1998.
} 
mostraba además de un friso rectangular con diversos animales un campo cuadrado, en el que se inscribía igualmente un gran círculo, decorado con otros círculos apenas visibles, y en los ángulos resultantes de la inscripción cuatro figuras de tritones, que sostenían con las manos alzadas el gran círculo central ${ }^{7}$.

No obstante, el paralelo más estrecho para el mosaico de Alcolea del Río lo encontramos en un mosaico de fecha similar que, datado también en la segunda mitad del siglo II d.C, fue hallado en Mitilene (Lesbos) (CHATZI, 1972, 588-591; ASEMAKOPOULOS, 1973, 239, $\mathrm{n}^{\circ}$ 43), en cuyo Nuevo Museo Arqueológico actualmente se conserva ${ }^{8}$. Apenas conocido ${ }^{9}$, el mosaico (fig. 3) fue descubierto durante las excavaciones realizadas entre el 9 de febrero y el 26 de abril de 1971, tras la noticia de hallazgos en el transcurso de las obras acometidas en la calle Potámonos, y pavimentaba originalmente una estancia, de 4,48 (este-oeste) por 3,73 m., de una casa romana. El pavimento contenía, de fuera a dentro, una banda ancha de teselas blancas, de $0,80 \mathrm{~m}$. de ancho en dos lados y de 0,40 en los otros dos, salpicada en el centro por una fila de crucecitas de teselas negras; a continuación, una banda de teselas negras delimitando un marco cuadrado, de 2,92 m. de lado, que aparecía bordeado además por una trenza y otro marco de teselas blancas y negras, y en el gran cuadrado que delimitaba el campo del mosaico la inscripción de un gran círculo de 2 metros de diámetro, decorado con el motivo de la roseta de triángulos curvilíneos, desarrollada en torno a un pequeño círculo central completamente lagunoso, mientras en tres de los ángulos resultantes de la inscripción del gran círculo en el campo cuadrado todavía se conservaban figuras de la mitología relacionadas con el mundo marino.

La roseta bícroma del mosaico de Mitilene está compuesta por 7 anillos concéntricos de 24 triángulos curvilíneos cada uno, siguiendo, por tanto, el trazado de una de las dos series más repetidas, aquella según la cual el mosaísta habría dividido la circunferencia en seis partes tomando como medida el radio y partiendo después los sectores (LUZON, 1988, 219). La representación de esta roseta en el mosaico de Mitilene avala además el

${ }^{7}$ El único tritón que se ha conservado, aunque no se expone en el Landesmuseum de Trier (SCHINDLER, 1986, 110), pertenece a la variedad de tritones de aletas natatorias y su figura joven e imberbe avanza, con el torso casi de frente y la cabeza de tres cuartos, hacia la derecha. Al figurar en esta posición y con las manos alzadas se inscribe entre los del tipo 5, que aún no siendo demasiado numerosos, se caracterizan por sostener sobre su cabeza en sentido horizontal un atributo, un remo o un kantharos (NEIRA, 1992, 170). En este sentido, llama la atención en el mosaico de Trier su papel, como soportes del círculo central, más propio de las figuras de gigantes como en un pavimento de Horkstow (PARLASCA, 1959, 55, nota 3).

${ }^{8}$ Queremos hacer constar aquí todo nuestro agradecimiento a Aglaia Archontidou-Argyri, Conservadora del citado Museo de Mitilene, quien nos ha brindado amablemente información sobre la localizacíón actual del mosaico, así como fotografías con detalles del mismo.

${ }^{9}$ Igualmente queremos manifestar en estas líneas nuestra gratitud a la Dra. Alicia Villar, Profesora de griego moderno, que nos ha prestado su ayuda en la traducción de las noticias dadas por D. Chatzi. 
seguimiento de unas reglas y unas medidas fijas en la aplicación de este motivo, ya que su inserción en el pavimento no se ha adecuado al espacio a pavimentar, sino que se ha reproducido según el modelo, acudiendo a bandas de mayor o menor anchura, las salpicadas por crucecitas, para completar la decoración de la superficie restante por cubrir.

En cuanto a las figuras marinas situadas en los ángulos, la que decoraba el inferior izquierdo -según la orientación marcada desde la puerta de acceso a la estancia, cuyo vano es apreciable en el extremo izquierdo del lado sur- contiene la representación de un anciano y barbado centauro marino (lám. 5), que, dotado de un par de antenas de crustáceo, sobresaliendo entre sus cabellos, avanza hacia la derecha, visto de tres cuartos, y, con los brazos fiexionados y hacia delante, sostiene con ambas manos un delfín. Pertenece al ya mencionado tipo 4 y concretamente a aquella serie de tritones que suelen flanquear a la Venus marina, sosteniendo los extremos de su concha (NEIRA 1992, 168 ss), aunque el paralelo más directo lo constituye la representación de un maduro y barbado tritón de aletas natatorias que aparece sosteniendo con ambas manos la figura de un Eros alado y desnudo en actitud de tocar la lira en un fragmentario mosaico bícromo de porta Collina, Roma (NEIRA, 1996, figs. 4-5).

En el ángulo inferior derecho destaca, en cambio, una representación de Scylla (lám. 6). La bella joven, que había sido transformada en un terrible monstruo marino de doce patas, seis cuellos gigantescos y seis cabezas con tres líneas de dientes (Hom. Od. 12, 80-97) a causa de los celos de Circe, en rivalidad por el amor de Glaucos (Ov. met. 13, 900-968; 14, 1-74; Hyg.fab. 199) o según otra versión por la envidia de Amphitrite al saberla amada por Poseidón, aparece, según una de las variantes de su iconografía más frecuente, como una figura de torso femenino, cuya parte inferior del cuerpo adopta una forma pisciforme con varias aletas y dos enroscadas colas finalizadas en cabeza de perro (JENTEL, 1997, 1145). Su torso figura casi de frente, ligeramente de tres cuartos hacia la izquierda, como si avanzara en esa dirección, aunque torna la cabeza, vista de tres cuartos, con los cabellos cayéndole sobre los hombros, y su mano izquierda hacia atrás, hacia el apéndice canino, al tiempo que en la derecha, alzada, sostiene el extremo de su característico timón de espadilla, dispuesto en sentido horizontal por detrás de su cabeza. En líneas generales, la Scylla del mosaico de Mitilene presenta las mismas características que el resto de las representaciones documentadas en la musivaria romana, aunque ella aparece sosteniendo la vara del timón con ambas manos alzadas tanto en los pavimentos bícromos de Tor Marancia (NOGARA 1910, lám. XXI) y las termas de Neptuno de Ostia (BECATTI, 1961, núm 71), como en los polícromos de Acholla (GOZLAN, 1974, fig. 58), Thaenae ${ }^{10}$ (MASSIGLI, 1912, láms. 11,1; 111,2 y IV,2), y

En este fragmentario mosaico, que pavimentaba el frigidarium de las termas de Thaenae, se documentan hasta tres medallones con la representación de Scylla. Dos presentan una gran similitud y responden al tipo 
Sila, (GSELL, 1905, lám. I; JENTEL, 1997, núm. 35) ${ }^{11}$. A este respecto, la representación de Mitilene es más similar a la de algunos relieves, como el de la coraza de una estatua hallada en el Agora de Atenas, de principios del siglo II d.C. (JENTEL, 1997, núms. 46-50, esp. 47).

Su papel, no obstante, en los mosaicos varía, ya que puede aparecer como figura central en torno a la cual se dispone un cortejo de tritones y/o nereidas, como en los mencionados de Ostia y Sila, o compartiendo protagonismo en diferentes esquemas compositivos con representaciones de la nave de Ulises, una nereida y otros animales marinos en Tor Marancia, con un gran cortejo de nereidas, tritones, divinidades fluviales y erotes sobre delfines en medallones dispuestos en torno a una representación central del Triunfo de Neptuno en Acholla, con infinidad de nereidas, tritones, la Venus marina y otras figuras relacionadas con la mitología marina en medallones hexagonales dispuestos en torno a la figura central de Arión en Thaenae, y de nuevo con tritones, erotes y Venus marina en Gubbio, por lo que no es de extrañar, aunque los ejemplos documentados hasta aquí sólo contenían en los ángulos figuras de tritones, que se haya incluido en este mosaico una representación de Scylla.

Además del mencionado tritón y Scylla, otra figura se conservaba aunque fragmentaria en el ángulo superior derecho. De ésta sólo restaba una sinuosa cola pisciforme que ascendía por el lado superior, así como un timón de espadilla dispuesto en sentido horizontal tras la cabeza y cuyo extremo debía sujetar con su mano derecha alzada. A juzgar por estos restos, podría tratarse de un tritón que, además de portar un timón de espadilla en su mano derecha, figuraría avanzando hacia la izquierda y volviendo ligeramente su cabeza hacia atrás, vista de tres cuartos, mientras su mano izquierda podría haber figurado en la cintura, como los pertenecientes al tipo 1 (NEIRA, 1992, 141 ss.), o más probablemente, extendida hacia atrás, como los del tipo 2 (NEIRA, 1992, 153-163). Sin embargo, tanto los tritones del tipo 1 como los del 2 no suelen aparecer representados con la mano - en la que portan un timón, remo o atributo similar por detrás de la cabeza - tan alzada, sino ligeramente adelantada, de modo que la vara del atributo en cuestión se dispone en sentido diagonal y no

citado, según el cual Scylla empuñaba con ambas manos la vara del timón en una actitud de ataque lógica ante la proximidad en los medallones colindantes de representaciones con la nave de Ulises, alusivas a la leyenda narrada en La Odisea; mientras la tercera, en cambio, muy dañada por una laguna, lo porta en sentido diagonal hacia un lado.

" Otra representación de Scylla se documenta también en un mosaico polícromo hallado en Gubbio (STEFANI, 1942, 372-373; NEIRA, 1992, n 71, lám. CLVIII), donde en un alargado friso fragmentario aparece en compañía de un eros alado y desnudo que cabalga de pie sobre un hipocampo, un tritón guiando las riendas de un toro marino y de otros tritones que sostenían la concha de la Venus marina. En este pavimento, Scylla se muestra igualmente bajo la forma de un torso femenino terminado en aletas de las que surgen, flanqueándola, dos perros, pero una laguna que afecta a su cabeza nos impide saber si portaba el característico timón de espadilla. 
tan horizontal como figura aquí y en la anteriormente mencionada representación de Scylla. En este sentido, dado, en primer lugar, que la laguna existente se cierne sobre el centro y la parte izquierda de la figura, - donde hay espacio para otro apéndice pisciforme - y en segundo lugar, que algunas de las citadas composiciones con figuras de tritones situadas en los ángulos responden a una similitud que puede reflejarse en los cuatro, como en los pavimentos ostienses de las Termas de Neptuno y las Termas Marítimas, o por parejas, como en Alcolea, cabría también la posibilidad de que, en lugar de un tritón, hubiera figurado aquí otra representación de Scylla, formando «pendant» con la ya citada (fig. 4). Ambas estaban orientadas al mismo lado, en cuyo muro interior y en la parte central se podía apreciar a $1,60 \mathrm{~m}$. sobre el suelo una cavidad semicircular de $0,95 \mathrm{~m}$. de longitud, 0.30 de anchura y $0,40 \mathrm{~m}$. de altura, revestida con una placa de mármol, según los datos proporcionados por la excavación (CHATZI, 1972, 588, fig. 11). Según esta hipótesis, es de suponer que otro tritón semejante al anciano y barbado centauro marino antes citado hubiera decorado el cuarto ángulo del campo, completamente afectado por una laguna ya en la época de su descubrimiento.

Son, por tanto, evidentes las similitudes que presentan los mosaicos de Alcolea del Río y Mitilene, en cuanto los dos muestran como motivo significativo la roseta bícroma de triángulos curvilíneos y la disposición sobre los ángulos de figuras marinas, sean tritones o tritones y representaciones de Scylla, que según nuestra hipótesis responderían por parejas a un carácter idéntico. A ello hay que añadir la combinación de bicromía - la roseta - y policromía - las figuras situadas en los ángulos, así como la cabeza de Medusa en Alcolea - que destaca en ambos pavimentos. Esta peculiaridad está bien documentada en la musivaria hispana de la segunda mitad del siglo II d.C, donde, como ejemplos más significativos puede citarse el llamado mosaico del triunfo de Neptuno de Itálica (BLANCO, LUZON, 1974), con una orla de tipo nilótico y un thiasos marino en teselas blancas y negras y una representación central con la travesía del dios del mar en policromía (NEIRA, 1996b, 567, tav. VII,2), o el mosaico emeritense del Rapto de Europa (BLANCO, 1978, núm. 4, lám. 5), donde en una composición geométrica de carácter bícromo se inserta un pequeño cuadro figurado con la leyenda del rapto en teselas de diversos colores. Asimismo la combinación de bicromía y policromía aparece en la musivaria romana de Grecia por la misma época (ASEMAKOPOULOS, 1973, 216-254; WAYWELL, 1979, 293-321), explicándose igualmente como una evolución propia del ámbito provincial, en el que junto a la influencia de la musivaria itálica de carácter bícromo se comienza a experimentar el uso de la policromía.

En esta línea, es muy significativa en los pavimentos romanos de Grecia la representación de la mencionada roseta de triángulos curvilíneos bícroma (PANAGIOTOPOULOU, 1994, 370-371, figs. 4, 6-7) en torno a un motivo central 
polícromo, tendencia en la que, a tenor de las figuras polícromas dispuestas sobre los ángulos del campo, se inserta el mosaico de Mitilene. A este respecto, sobre el motivo o figura que habría decorado en origen el círculo central, no sólo la similitud con el pavimento de Alcolea, sino también la frecuencia con la que aparece como motivo central de una roseta de triángulos curvilíneos en los ya citados mosaicos de Carmona (BLAZQUEZ, 1982), Museo Paul Getty de Malibú (VERMEULE, NEUERBURG, 1973, núm. 111), Roma (Emmanuele Huberto) y Ostia (LUZON 1988, núms. 1415), entre otros, y especialmente en mosaicos de Grecia, procedentes de Cnossos, Patras y Thasos (PANAGIOTOPOULOU, 1994, 370-371) nos llevan a pensar en una cabeza de Medusa.

La representación de la Medusa cuenta además con gran tradición en la musivaria de Grecia, donde existen al menos una veintena catalogadas (PANAGIOTOPOULOU, 1994, 369-383), de las que 14 figuraban en el centro del campo, decorando el círculo en torno al cual se disponía una roseta de triángulos o de escamas, tanto bícromas como polícromas. Inicialmente como representación de la Medusa situada en el escudo de Atenea, quien la habría colocado allí tras recibirla del victorioso Perseo, Medusa (KRAUSKOPF, 1988, 285-330; PAOLETTI, 1988, 345-362) será un símbolo tradicionalmente apotropaico, que, aún perdiendo progresivamente sus rasgos demoníacos, pervivirá en época romana. En este sentido, la representación de su rostro orientado hacia la entrada de la estancia es frecuente en la musivaria romana (McKEON, 1986) si bien es posible que acabara por convertirse en un motivo común del repertorio decorativo (DUNBABIN, 1978, 163).

Su inclusión en el mosaico de Alcolea del Río, - vista casi de frente y ligeramente de tres cuartos hacia la izquierda, dotada del característico par de alitas sobresaliendo entre sus dorados cabellos y mostrando un rostro afable -y probablemente en Mitilene en unión de representaciones tan significativas como las de los tritones y Scylla refuerza no obstante ese carácter apotropaico, que nos inclina a interpretar la decoración de ambos pavimentos como un símbolo conscientemente incorporado para evitar los malos espíritus y procurar al tiempo protección y buena fortuna a los habitantes de la casa.

\section{BIBLIOGRAFÍA}

ASEMAKOPOULOS, G. (1973): «Catálogo de los mosaicos romanos de Grecia» (en griego), ELLHNIKA, 216-254, láms. 4-32.

AURIGEMMA, S. (1958): Le Terme di Diocleziano e il Museo Nazionale Romano, Roma (4a. ed.). 
BARRAL i ALTET, X. (1978): Les mosdiques romaines et medievals de la Regio Laietana, Barcelona.

BECATTI, G. (1961): Scavi di Ostia IV Mosaici e pavimenti marmorei, Roma.

BENDALA, M. (1976): La Necrópolis romana de Carmona (Sevilla), Sevilla.

BLAKE, M.E. (1940): «Mosaics of the late Empire in Rome and vicinity», MAAR XVII, 95.

BLANCO, A. (1978): Mosaicos romanos de Mérida. Corpus de Mosaicos de España I, Madrid.

(1978a): Mosaicos romanos de Itálica. Corpus de Mosaicos de España II, Madrid, 8, 32-34, fig. 2, láms. 20-27.

BLANCO, A., LUZON, J.M . (1974): El mosaico de Neptuno en Itálica, Sevilla. BLAZQUEZ, J.M. (1981): Mosaicos romanos de Córdoba, Jaén y Málaga. Corpus de Mosaicos de España III, Madrid.

BLAZQUEZ, J.M. (1982): Mosaicos romanos de Sevilla, Granada, Cádizy Murcia, Corpus de Mosaicos de España IV, Madrid.

BONSOR, G.E. (1901): «Los pueblos antiguos del Guadalquivir y las alfarerías romanas», RABM, 837-857.

(1931): The Archaeological Expedition along the Guadalquivir 18891901, Nueva York.

BROISE, H. ET ALII (1977): «Eléments antiques sitúes sous le Palais Farnése», MEFRA 89, 723-797, esp. 785-797

CAMPOS, M. de (1897): Mosaicos del Museo Arqueológico Provincial de Sevilla, Sevilla.

CHATZI, D. (1972): «Crónica», Archaelogicon Deltion 27, 588-591, figs. 11-12, láms. 535, (en griego).

DARMON, J.P. (1980): Nympharum Domus. Les pavements de la maison des Nymphes á Neapolis (Nabeul, Tunisie) et leur lecture, Leiden.

FOUCHER, L. (1965): «Les mosai'ques nilotiques africaines», CMGR I, 142, fig. 3.

GALI, A. (1892): Historia de Itálica. Municipio y colonia romana, Sevilla.

GOZLAN, S. (1974): «Les pavements en mosaïques de la Maison de Neptune á Acholla-Botria (Tunisie)», MonPiot 59, 112-135. 
GSELL, S. (1905): «Mosaíque romaine de Sila», Rec. Constantine XXXIX, 1-7, lám. I.

TheHispanicSociety of America. Handbook. MuseumandLibrary Collections (1938): Nueva York.

JENTEL, M.O. (1997), «Skylla I», LIMC VIII/1-2, 1997, 1137-1145.

KORSUNSKA, S. (1928): « Römischen Mosaiken in Zarskoje Sselo», JDAI43, 360371, fig. 1,lám. 12.

KRAUSKOPF, I. (1988): «Gorgo, Gorgones», LIMC IV, 293-330.

LANCIANI, R. (1885): «Note sopra le scoperte avvenute durante il mese di settembre. Roma, regio II. Villa Casali» NotScavi, 341, 422.

LUZON, J.M. (1988): «La roseta de triángulos curvilíneos en el mosaico romano», Anejos de Gerión I. Homenaje a García Bellido V, 213-241.

McKEON, C.H. (1986): Iconology ofthe Gorgon Medusa in Román Mosaic, Michigan, Tesis Doctoral Universidad de Michigan.

MAIER, J. (1997): «Las Sociedades Arqueológicas en España: La Sociedad Arqueológica de Carmona», en MORA, G. y DÍAZ ANDREU, M. (eds.), La Cristalización del Pasado: Génesis y Desarrollo del Marco Institucional de la Arqueología en España, Málaga, 303-310.

(1997a): Jorge Bonsor (1855-1930). Personalidady significación de un pionero de la Arqueología. II Epistolario, Madrid Tesis Doctoral Universidad Autónoma de Madrid.

MAIURL A. (1958): Ercolano. I nuovi scavi (1927-1958) I, Roma.

MASSIGLI, R. (1912): Museés de l'Algérie et te la Tunisie. Musée de Sfax, París.

MORETTI, G. (1925): «Sassoferrato. Mosaico romano», NotScavi, 110-113:

NEIRA, M.L. (1991): «Acerca de las representaciones de thiasos marino en los mosaicos romanos tardo-antiguos de Hispania», Antigüedad y Cristianismo VIII, 513-529.

(1992): La representación del thiasos marino en los mosaicos romanos. Nereidas y tritones, Madrid Tesis Doctoral Universidad Complutense de Madrid.

(1994): «Mosaico de los tritones de Itálica en el contexto iconográfico del thiasos marino en Hispania», VI CIMA, 359-367.

(1996): «Representación de thiasos marino en tres fragmentos de mosaico bícromo», Homenaje a José M. Blázquez III, Madrid, 223-249, figs. 1-9. 
(1996b): «La tipología del carro en los mosaicos romanos del triunfo de Neptuno», L'Africa romana XI, 555-576.

NOGARA, B. (1910): Imosaici antichi conservan nei Palazzi Apostolici del Vaticano e nel Luterano, Milán.

OVADIAH, A. (1980): Geometric and Floral Patterns in Ancient Mosaics, Roma. PACKARD, P.M. (1980): «A monochrome Mosaic at Isthmia», Hesperia 49, 326346.

PANAGIOTOPOULOU, A. (1994): «Représentations de la Meduse dans les mosaiques de Gréce», VI CIMA, 369-383.

PAOLETTI, O. (1988): «Gorgones Romanae», LIMC IV, 345-362.

PARLASCA, K. (1959): Römische Mosaiken in Deutschland, Berlín.

SCHINDLER, R. (1986): Führer durch das Landesmuseum Trier, Trier.

STEFANI, E. (1942): «Resti di un'antica costruzione con pavimento a mosaico lungo la via di S. Biagio», NotScavi, 372-373.

THEODOROU, J. (1991): «Treasures of Ancient Spain, The Hispanic Society of America», MINERVA vol. 2, núm. 6, nov.-dec, 33, fig. 6.

TOMASE VIC, G.C. (1975): «Mosaiques paléochrétiennes récemment découvertes á Héracléa Lynkestis», CMGR II, 385-399.

VERMEULE, C, NEUERBURG, C. (1973): Catalogue of the Ancient Art in the J. Paul Getty Museum, Malibú.

WAYWELL, S.E. (1979): «Roman Mosaics in Greece», AJA 83, 293-321. 


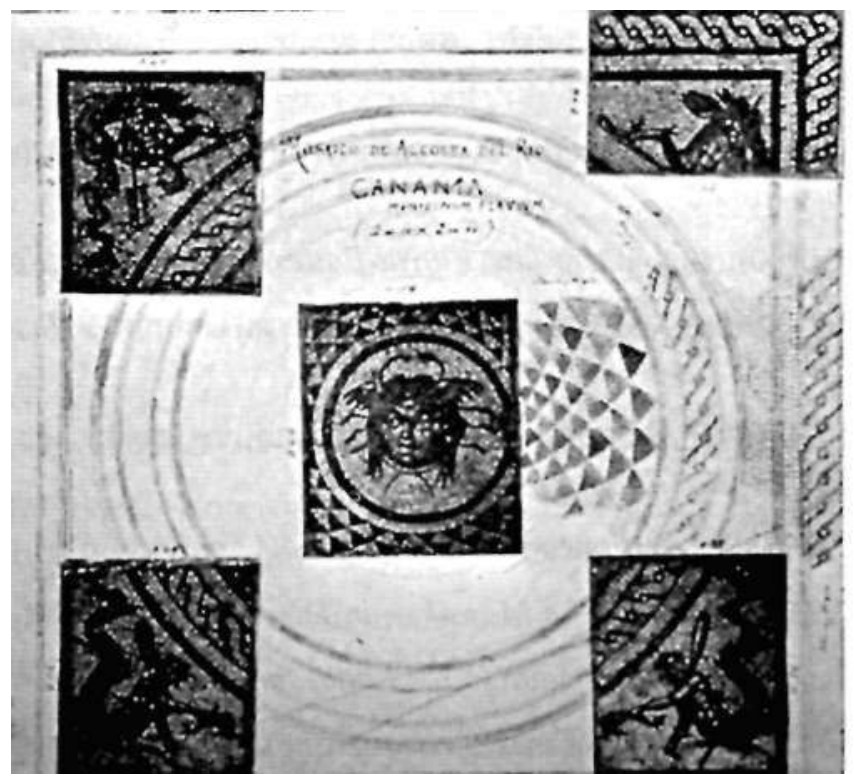

Fig. 1: Reconstitución del mosaico de Alcolea del Río (Sevilla), según Bonsor.
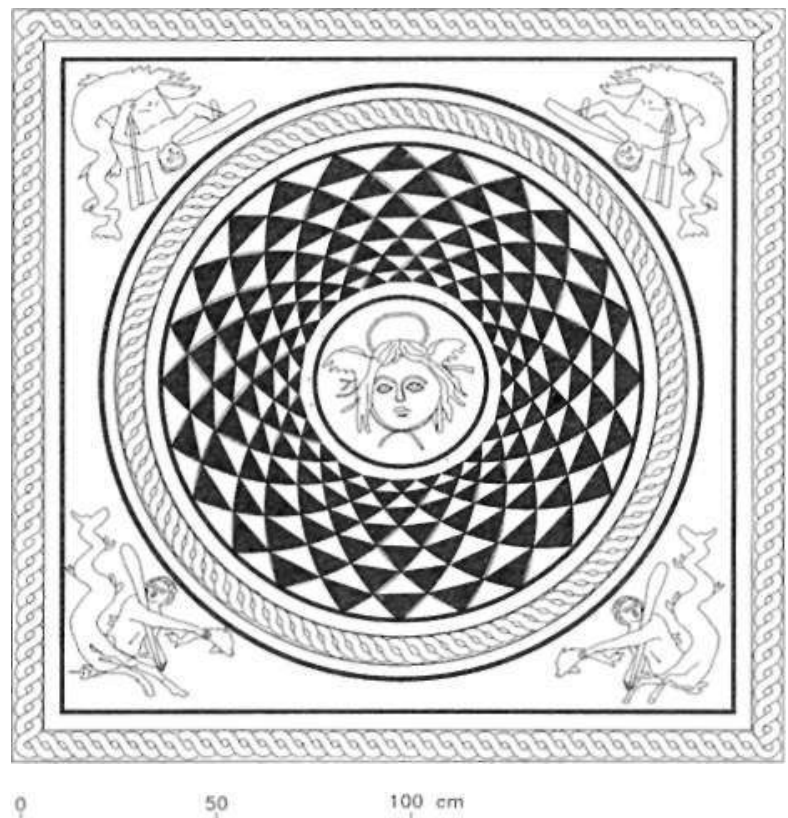

$100 \mathrm{~cm}$

Fig. 2: Mosaico de Alcolea del Río (Sevilla). Reconstitución de Luz Neira. Dibujo: Esteban Moreno (C.S.I.C.) 


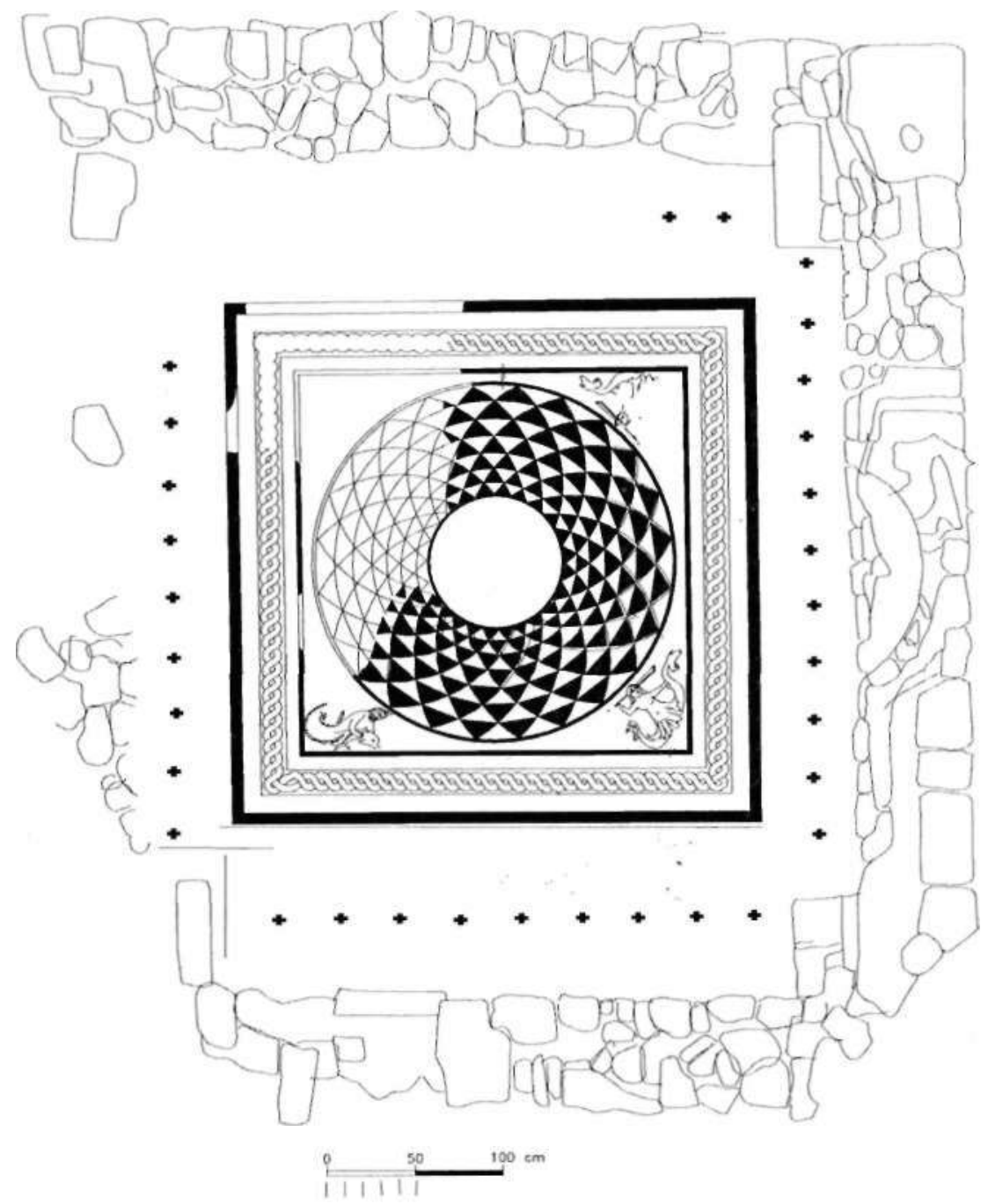

Fig. 3: Mosaico de Mitilene en el momento de su descubrimiento por Chatzi. Dibujo: Esteban Moreno (C.S.I.C.) 


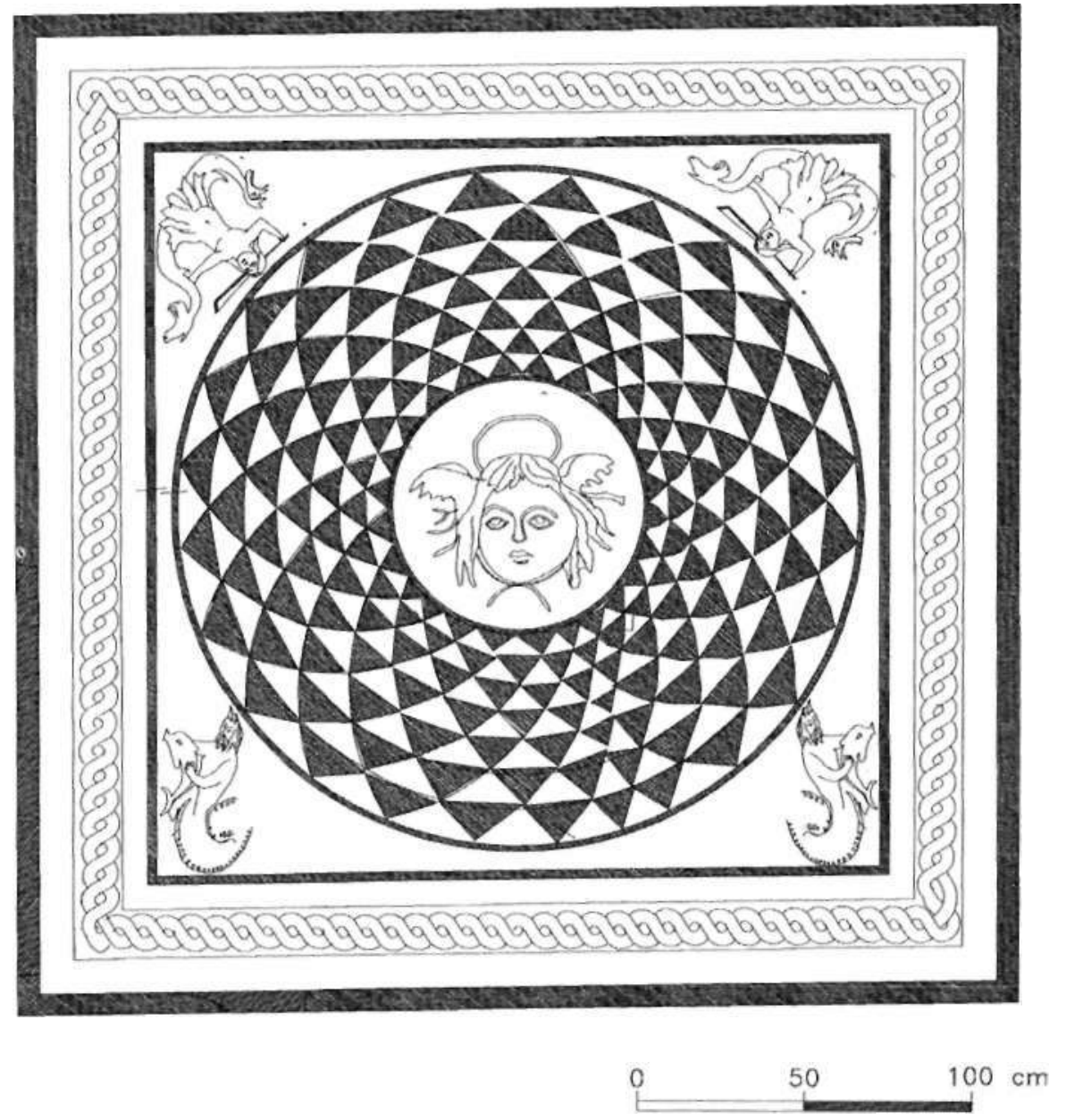

Fig. 4: Recosntruccion del mosaico de Mitilene según Luz Neira. Dibujo: Esteban Moreno (C.S.I.C.) 
Lam. 1: Fragmento central con la cabeza de Medusa. Foto del autor.
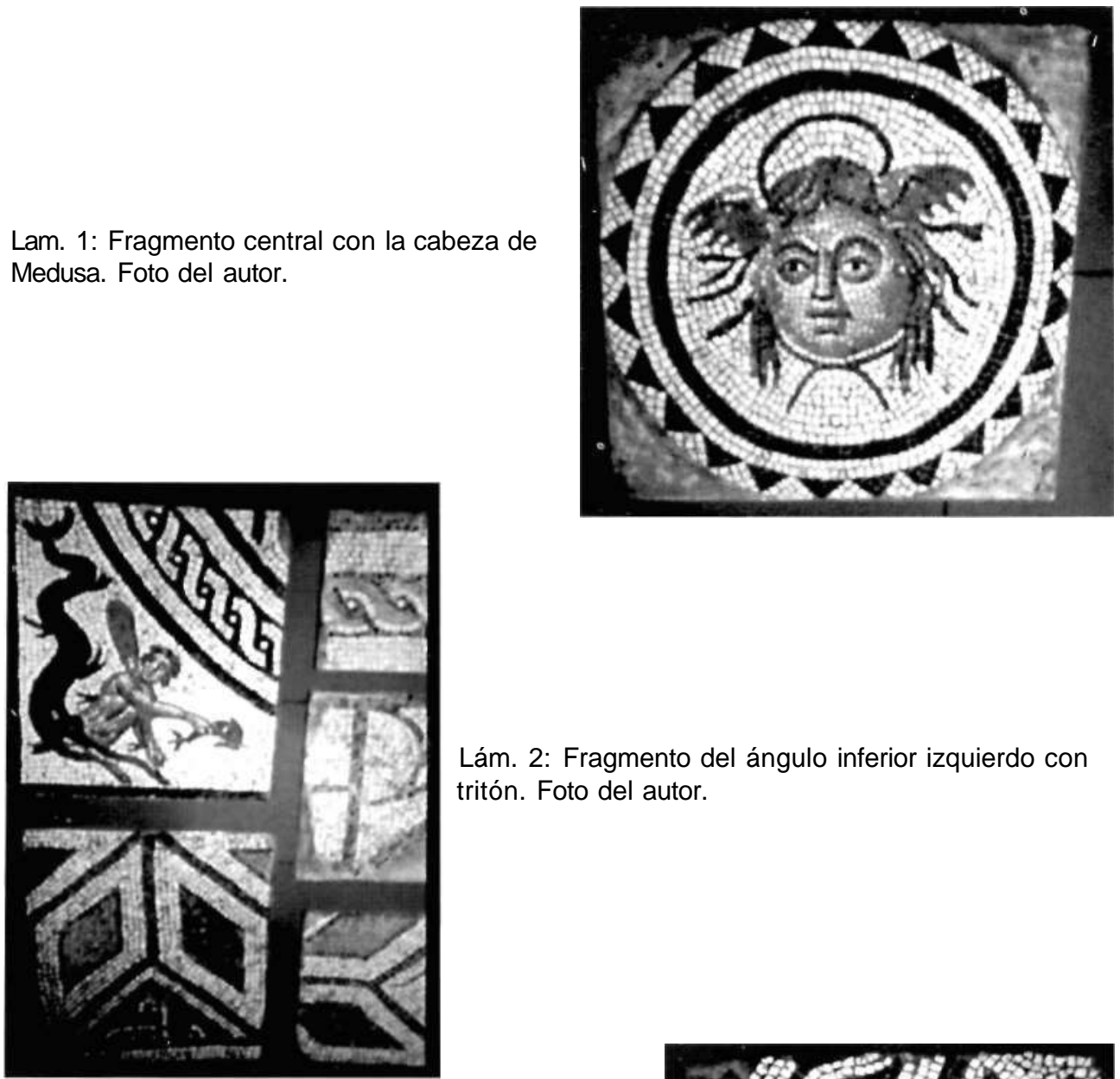

Lám. 2: Fragmento del ángulo inferior izquierdo con tritón. Foto del autor.

Lám. 3: Fragmento del ángulo inferior derecho con tritón. Foto del autor.

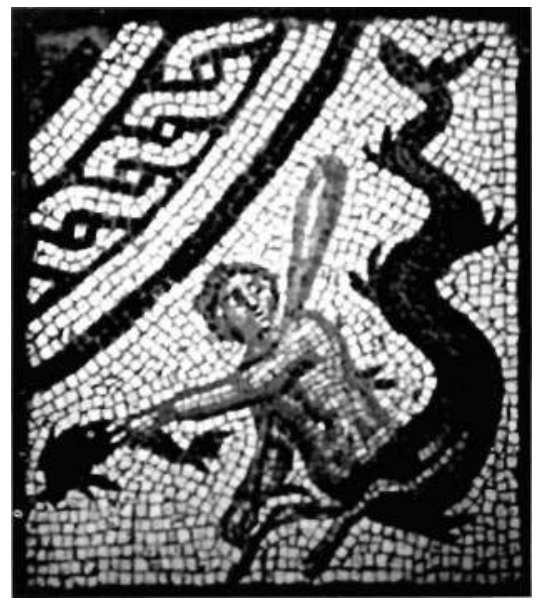




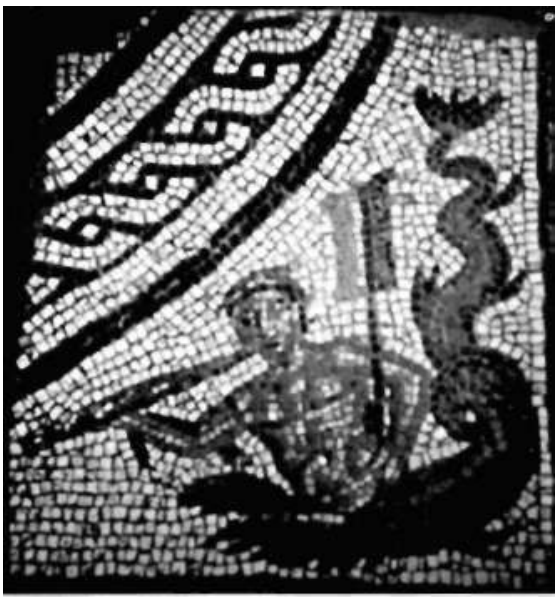

Lám. 4: Fragmento del ángulo superior izquierdo. Foto del autor.

Lám. 5: Mosaico de Mitilene. Detalle del tritón. Foto: Cortesía del Museo Arqueológico de Mitilene (Lesbos).
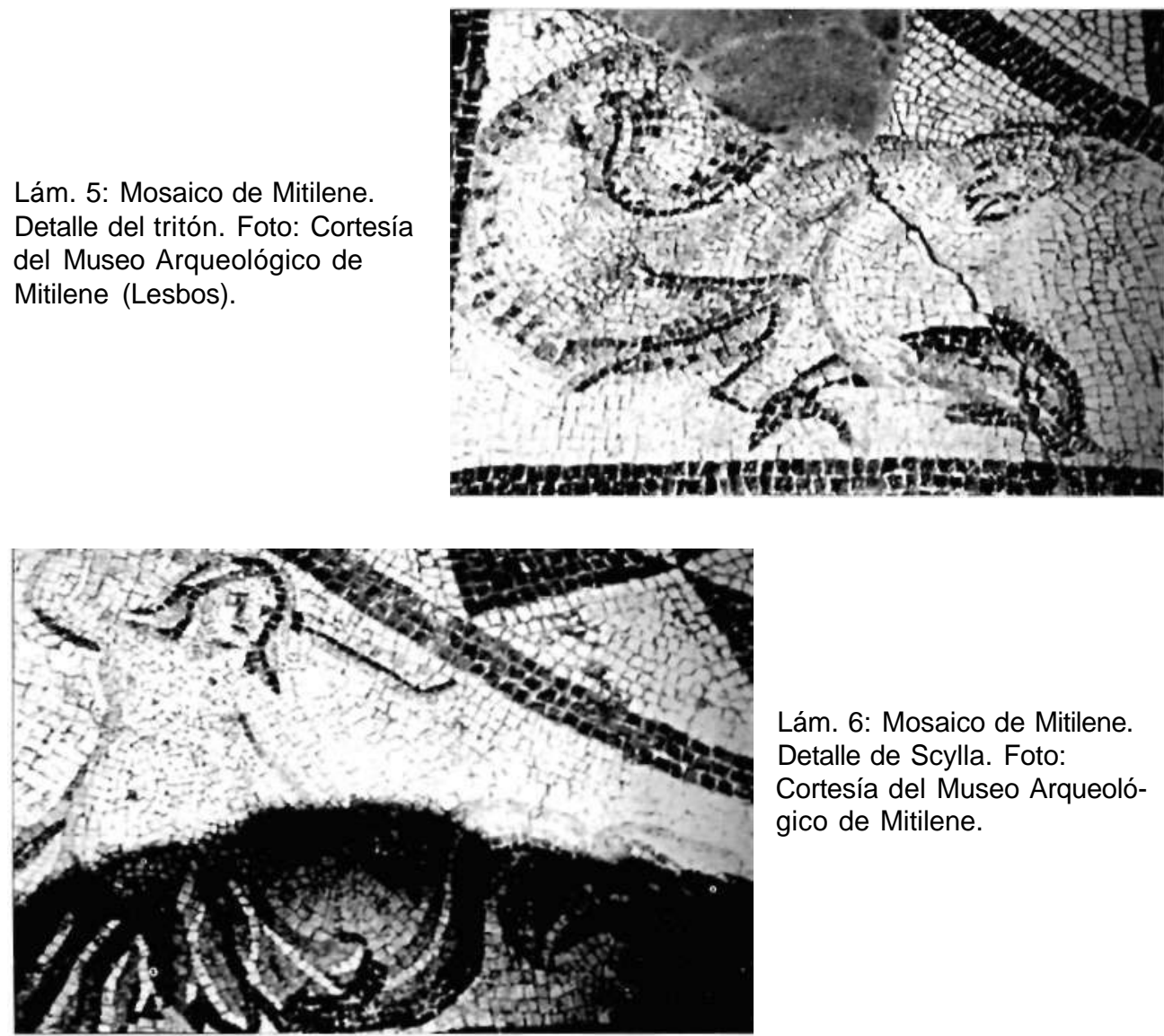

Lám. 6: Mosaico de Mitilene. Detalle de Scylla. Foto: Cortesía del Museo Arqueológico de Mitilene. 